\title{
Griscelli syndrome restricted to hypopigmentation results from a melanophilin defect (GS3) or a MYO5A F-exon deletion (GS1)
}

\author{
Gaël Ménasché, ${ }^{1}$ Chen Hsuan Ho, ${ }^{1}$ Ozden Sanal, ${ }^{2}$ Jérôme Feldmann, ${ }^{1}$ Ilhan Tezcan, ${ }^{2}$ \\ Fügen Ersoy, ${ }^{2}$ Anne Houdusse, ${ }^{3}$ Alain Fischer, ${ }^{1}$ and Geneviève de Saint Basile ${ }^{1}$
${ }^{1}$ Unité de Recherche sur le Développement Normal et Pathologique du Système Immunitaire, Institut National de la Santé et de la Recherche Médicale (INSERM) U429, Hôpital Necker-Enfants Malades, Paris, France
${ }^{2}$ Hacettepe Children's Hospital, Immunology Division, Ankara, Turkey
${ }^{3}$ Équipe Motilité Structurale, Centre National de la Recherche Scientifique UMR144, Institut Curie, Paris, France

\begin{abstract}
Griscelli syndrome (GS) is a rare autosomal recessive disorder that associates hypopigmentation, characterized by a silver-gray sheen of the hair and the presence of large clusters of pigment in the hair shaft, and the occurrence of either a primary neurological impairment or a severe immune disorder. Two different genetic forms, GS1 and GS2, respectively, account for the mutually exclusive neurological and immunological phenotypes. Mutations in the gene encoding the molecular motor protein Myosin $\mathrm{Va}(\mathrm{MyoVa})$ cause GS1 and the dilute mutant in mice, whereas mutations in the gene encoding the small GTPase Rab27a are responsible for GS2 and the ashen mouse model. We herein present genetic and functional evidence that a third form of GS (GS3), whose expression is restricted to the characteristic hypopigmentation of GS, results from mutation in the gene that encodes melanophilin (Mlph), the ortholog of the gene mutated in leaden mice. We also show that an identical phenotype can result from the deletion of the MYO5A F-exon, an exon with a tissue-restricted expression pattern. This spectrum of GS conditions pinpoints the distinct molecular pathways used by melanocytes, neurons, and immune cells in secretory granule exocytosis, which in part remain to be unraveled.
\end{abstract}

J. Clin. Invest. 112:450-456 (2003). doi:10.1172/JCI200318264.

\section{Introduction}

Griscelli syndrome (GS; MIM 214450) is a rare autosomal recessive disorder that results in a characteristic pigmentary dilution of the skin and the hair, with the presence of large clumps of pigment in hair shafts and an abnormal accumulation of end-stage melanosomes in the center of melanocytes (1-4). So far, two forms of GS have been described. Type 1 (GS1) associates characteristic albinism with a severe primary neurological impairment. Patients exhibit severe developmental delay and mental retardation occurring early in life. These patients carry mutations of the myosin 5A gene (MYO5A) (2), which encodes an organelle motor protein, Myosin Va (MyoVa), with a determining role in neuron function

Received for publication March 3, 2003, and accepted in revised form May 13, 2003.

Address correspondence to: Geneviève de Saint Basile, INSERM U429, Hôpital Necker-Enfants Malades, 149 rue de Sèvres, 75015 Paris, France. Phone: 33-1-44-49-50-80; Fax: 33-1-42-73-06-40; E-mail: sbasile@necker.fr. Chen Hsuan Ho and Ozden Sanal contributed equally to this work.

Conflict of interest: The authors have declared that no conflict of interest exists.

Nonstandard abbreviations used: Griscelli syndrome types 1, 2, and 3 (GS1, GS2, and GS3); Myosin Va (MyoVa); hemophagocytic syndrome (HS); melanophilin (Mlph); Slp homology domain (SHD); patients $\mathrm{A}$ and $\mathrm{B}$ (PA and $\mathrm{PB})$.
(5). The second type of Griscelli syndrome (GS2) is characterized by the same hypopigmentation associated with an immune defect, leading to episodes of a life-threatening uncontrolled $\mathrm{T}$ lymphocyte and macrophage activation syndrome known as accelerated phase or hemophagocytic syndrome (HS) (6). During HS, activated T cells and macrophages infiltrate various organs (including the brain), leading to massive tissue damage, organ failure, and death in the absence of an immunosuppressive treatment. Bone marrow transplantation is the only curative treatment for this condition (6). Mutations in RAB27A, encoding a small GTPase protein (Rab27a) involved in the function of the intracellular-regulated secretory pathway, cause GS2 (3). The immune deregulation observed in GS2 patients is accounted for by the absolute requirement of the Rab27a function in lymphocyte cytotoxic granule release and the determining role of this cytotoxic pathway in immune homeostasis $(3,7,8)$. Both genes (MYO5A and $R A B 27 A$ ) map to the same chromosome $15 \mathrm{q} 21.1$ region and are distant from each other by less than $1.6 \mathrm{cM}(3,9)$.

Natural murine models of GS1 and GS2 have been respectively described as dilute (Myo5a-) (10) and ashen $($ Rab27a-) mice (11). These mutants present a phenotype close to that of their human counterparts, although HS has not so far been described in ashen 
mice (10-13). An identical pigmentary dilution and melanosome transport defect has been described in leaden mice, resulting from a mutation in melanophilin $(M l p h)$, which encodes a member of the Rab effector family (14). The protein complex formed by Rab27a, Mlph, and MyoVa has been recently shown to be essential for the capture and local movement of melanosomes in the actin-rich cell periphery of melanocytes (15-17). Mlph belongs to the Slp (synaptotagmin-like protein)/Slac2 (Slp homolog lacking C2 domains) family. Several members of this family have been reported to act as effectors of Rab27a. They directly bind Rab27a via their $\mathrm{N}$-terminal Slp homology domain (SHD). In addition to this, biochemical analyses have indicated that Mlph, via its C-terminal domain, interacts with $\mathrm{MyoVa}$ (16).

In this report, we show, for the first time to our knowledge, that homozygous missense mutation in human melanophilin $(M L P H)$, leading to defective Rab27a-Mlph interaction, results in a third form of GS (GS3), the phenotype of which is restricted to hypopigmentation. In addition, we report that this same pigment-dilution phenotype can also occur as a result of MYO5A F-exon deletion. These findings provide further insight into the understanding of the expression spectrum of GS and further strengthen the hypothesis that Rab27a acts in the secretory pathway through different groups of effectors in different cell types.

\section{Methods}

The clinical presentation of patients $\mathrm{A}$ and $\mathrm{B}$ (PA and $\mathrm{PB}$ ) has been previously reported (respectively, P12 and P11 in ref. 18). PA and PB are unrelated; both belong to consanguineous families. PA was first referred at the age of 10 years with the complaint of failure to gain weight, while PB was referred at the age of 4 years because of recurrent tonsillitis. In both patients, silver-gray hair, eyebrows, and eyelashes were noticed. Microscopic analysis of their hair shafts showed the characteristic features of Griscelli syndrome, i.e., the presence of large clumps of pigment in the hair shaft (Figure 1). A longitudinal follow-up of each patient (over 6 and 8 years, respectively) has revealed that phenotypic presentation in both cases was restricted to hypopigmentation, without any immune or neurological manifestations. Growth was normal in PA. Informed consent for the study was obtained from the parents of the patients.

Genotype analysis and mutation detection. Genomic DNA was extracted from peripheral blood cells (19), and genotype analysis was performed as previously described (9), using known chromosome $15 \mathrm{q} 21$ markers spanning the RAB27A/MYO5A locus $(3,9)$, as well as markers neighboring the $M L P H$ gene locus on chromosome 2q37.3 (D2S2348, D2S338, D2S125, and D2S140) (20).

Mutational analysis of RAB27A (GenBank accession no. U38654; http://www.ncbi.nlm.nih.gov/Genbank/) and MYO5A (accession no. U90942) was performed as previously described $(3,9)$. Each exon of $M L P H$ (accession no. NM024101) was amplified on genomic DNA and sequenced directly using the ABI PRISM dye terminator (Applied Biosystems, Courtaboeuf, France). Sequence was determined from both strands. Primer sequences are available on request.

Molecular cloning of WT and mutants of Mlph. Cloning of WT human RAB27A in pFLAG-CMV-4 has been previously reported (21). A cDNA encoding the first 146 amino acids of human Mlph, termed Slp homology domain (SHD) (22), was cloned in frame into pcDNA3.1/Myc-His (Invitrogen, Cergy Pontoise, France), which adds the Myc epitope to the $C$ terminus of the cloned cDNA, as previously described (21). For mutant $M L P H$ constructs, site-directed mutagenesis (in boldface in the sequences) of $M L P H$ was performed using a double-PCR strategy (23). The primers used are as follows: 5'-CGAAGGAAAGAAGAGGAATGGCTAGAGGCGTTGAAG-3' (R35W primer, sense), 5'CTTCAACGCCTCTAGCCATTCCTCTTCTTTCCTTCG-3' (R35W primer, antisense), 5'-CGAAGGAAAGAAGAGGAAAAGCTAGAGGCGTTGAAG-3' (R35K primer, sense), 5'-CTTCAACGCCTCTAGCTTTTCCTCTTCTTTCCTTCGGAGGTCAAAATCTCG-3' (R35K primer, antisense), 5'-CGAAGGAAAGAAGAGGAAGTGCTAGAGGCGTTGAG-3' (R35V primer, sense), 5'-CTTCAACGCCTCTAGCACTTCCTCTTCTTTCCTTCGGAGGTCAAAATCTCG-3' (R35V primer, antisense), 5'-CGAAGGAAAGAAGAGGAATTGCTAGAGGCGTTGAAG-3' (R35F primer, sense), 5'-CTTCAACGCCTCTAGCAATTCCTCTTCTTTCCTTCGGAGGTCAAAATCTCG-3' (R35F primer, antisense).
Control

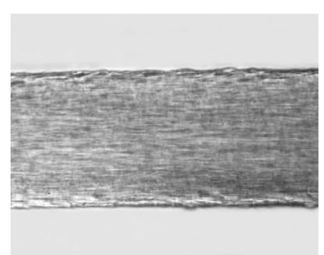

GS1

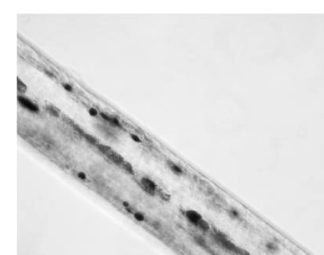

GS2

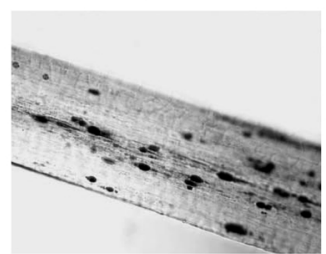

PB: GS1

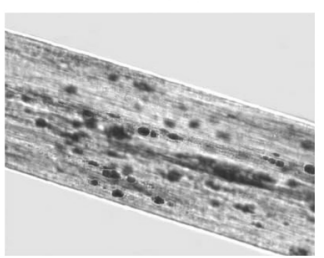

PA: GS3

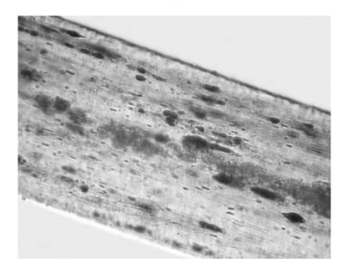

\section{Figure 1}

Light microscopy of patients' hair shafts. Typical features of GS are the large clumps of pigment irregularly distributed along the hair shaft, as shown for a GS1 patient and a GS2 patient. The same aspect is observed in the hair shafts of PA and PB. In contrast, a fine, evenly distributed pigment is observed in the control hair shaft. 


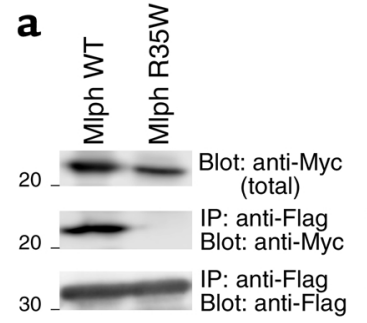

b

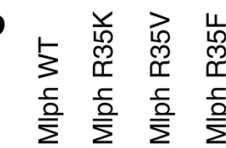
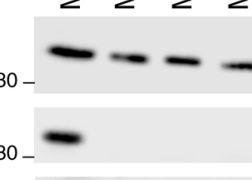

Blot: anti-Flag (total)

IP: anti-Myc
$20-$ Blot: anti-Flag

IP: anti-Myc Blot: anti-Myc

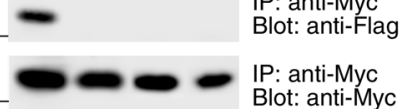

\section{Figure 4}

Association of Mlph mutant proteins with Rab27a. (a) MLPH mutation in PA affects Mlph-Rab27a interaction, as shown by coimmunoprecipitation analysis. WT Mlph (the MLPH sequence encoding the first 146 amino acids [SHD], cloned in pcDNA 3.1/Myc vector) or the mutant Mlph R35W, and Rab27a (the entire sequence encoding Rab27a, cloned in pFlag-CMV-4 vector), were cotransfected into $293 \mathrm{~T}$ cells, immunoprecipitated with an anti-Flag antibody, and revealed with an anti-Myc antibody (IP: anti-Flag; Blot: anti-Myc). A similar amount of proteins was present (top and bottom panels). The positions of the marker $\left(\times 10^{-3}\right)$ are shown on the left side. (b) None of the new substitutions introduced at position 35 of Mlph could restore Mlph-Rab27a interaction. The R35K, R35V, and R35F mutants were introduced in the SHD of Mlph by directed mutagenesis. Coimmunoprecipitated Flag-Rab27a detected by the anti-Flag antibody (IP: anti-Myc; Blot: anti-Flag) was only observed with the WT Mlph. The same blot was then stripped and reprobed with an anti-Myc antibody. The top panel indicates the total amount of expressed Flag-Rab27a (1:100 volume of the reaction mixture) used for immunoprecipitation. None of the mutant constructs was able to restore binding to Rab27a.

Association of Mlph mutant proteins with Rab27a. The protein complex formed by Rab27a-Mlph and MyoVa allows the melanosomes to connect to the actin network (27). In this complex, Mlph interacts with Rab27a through its N-terminal part (SHD) and with MyoVa through its C-terminal part. To further determine whether or not the R35W substitution identified in PA is sufficient to impair Rab27a-Mlph interaction, an $\mathrm{SHD}(\mathrm{R} 35 \mathrm{~W})-\mathrm{Mlph}$ construct was used to express the R35W mutant. It was tested in an in vivo Rab27a-binding experiment. Although SHD(WT)-Mlph coimmunoprecipitated with Rab27a when both constructs were coexpressed in the 293T cell line, the R35W mutation introduced in the SHD of Mlph completely blocked interaction with Mlph (Figure 4a). Since the arginine residue (R35) is exposed to the interface of the Mlph-Rab27a interaction, the replacement of this positively charged side chain residue $(\mathrm{R})$ by a residue with a bulky aliphatic side chain (W) may have affected the stereochemical

\section{Figure 5}

Overexpression of WT-SHD Mlph but not R35W-SHD Mlph interferes with melanosome transport. Melan-a cells were transfected with plasmids encoding PEGFP-C2, which allows a soluble GFP expression (a), or with GFP-WT-SHD-Mlph (c) or GFP-R35W-SHDMlph (e). (b, d, and $\mathbf{f}$ ) Images of transmitted light showing the melanosome distribution. Bars: $20 \mu \mathrm{m}$.

constraint of the interaction. The specificity of the R35 residue (as well as the biochemical basis of the Mlph-Rab27a functional interaction) was then further investigated by the analysis of additional substitutions at this very same position. Three substitutions ( $\mathrm{V}, \mathrm{F}$, and $\mathrm{K}$ in place of $\mathrm{R}$ ) were chosen because they would not disrupt protein folding but rather would introduce either a similar lateral chain size (V and F) or both a similar size and charge $(\mathrm{K})$ as compared with the arginine residue. When tested in the same conditions as WT Mlph, none of these mutant constructs, including the most closely related (K) substitution, could restore Mlph-Rab27a interaction (Figure $4 \mathrm{~b}$ ). These results highlight the specific requirement of the conserved $\mathrm{R}$ residue at position 35 for Rab27a/Mlph binding and confirm the deleterious effect of R35W mutation on MlphRab27a interaction.

Overexpression of Mlph R35 mutant does not modify melanosome distribution. We then examined the intracellular distribution of melanosomes as a function of the expression of either the WT-SHD or the R35W-SHD Mlph in melanocytes. Melan-a cells, a WT mouse melanocyte cell line, were found to exhibit evenly distributed melanosomes throughout the cell body (Figure 5, a and b). Transitory transfection of WT-SHD Mlph in Melan-a cells resulted in a dramatic redistribution of the pigment granules, which, in the majority of the transfected cells, became clustered in the perinuclear region. SHD Mlph can interact with the endogenous Rab27a, but truncation of the Mlph C-terminal part precludes targeting of MyoVa to the melanosomes, thus leading to a dominant negative effect mediated by this
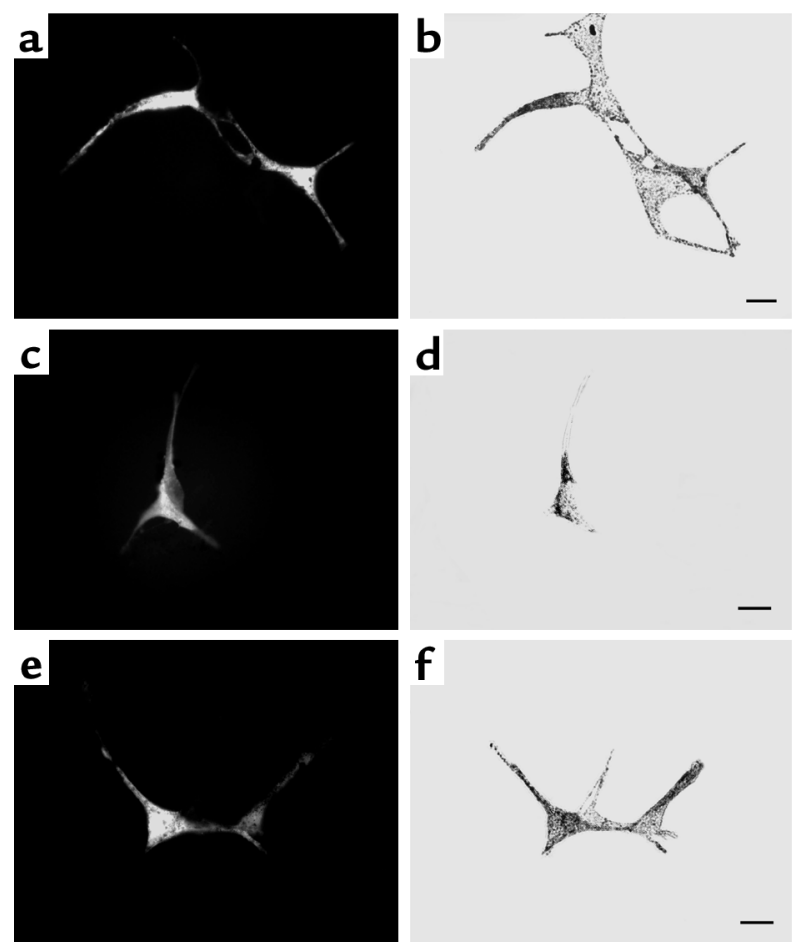

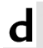

d
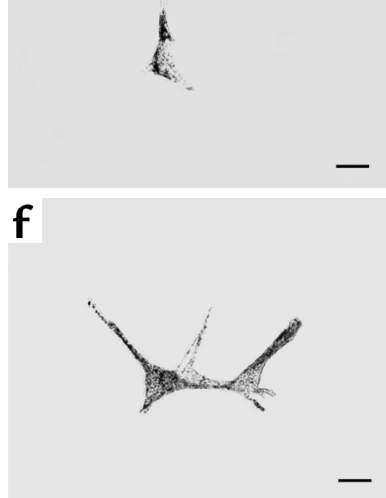


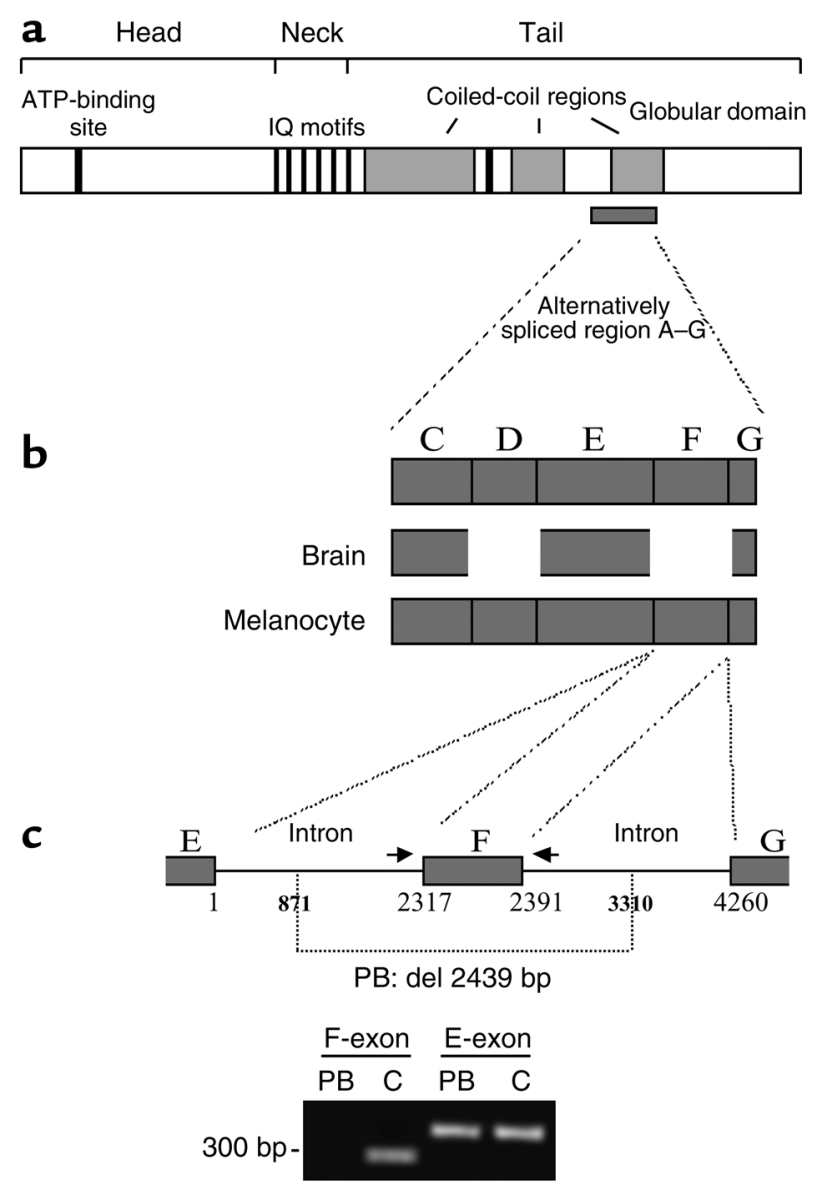

construct. In contrast to this, no effect on granule positioning was observed when the SHD Mlph mutant (R35W) was overexpressed in these cells (Figure 5, e and f); this confirmed the inability of the R35W mutant to interact with endogenous Rab27a. GS with a pure albinism phenotype caused by F-exon MYO5A gene mutation. Since PB displayed a GS phenotype identical to that of $\mathrm{PA}$, i.e., disease expression restricted to hypopigmentation, a similar genetic analysis was undertaken. Using segregation linkage analysis, the $M L P H$ locus was excluded as a potential candidate region in this family, while the RAB27A/ $M Y O 5 A$ locus was found compatible (data not shown). Mutation screening of the two causative genes of GS located in this region was thus undertaken. No mutation could be identified in the RAB27A gene, but sequencing of MYO5A in PB identified a homozygous 2,439-bp genomic deletion (del 871-3310) spanning the whole F-exon, as well as part of the flanking $5^{\prime}$ and $3^{\prime}$ intronic sequences (Figure 6, a-c). Several isoforms of MYO5A have been described, in both mice $(28,29)$ and humans $(9,30)$. They result from different combinations of spliced exons, along with the F-exon, which encode part of the tail region of MyoVa (Figure 6, a and b). The majority of MYO5A transcripts in melanocytes contain the F-exon, whereas brain transcripts lack the

\section{Figure 6}

Characterization of the MYO5A mutation in PB. (a) Schematic representation of MyoVa with the details of the various domains as previously reported (9) and of the alternatively spliced A-G region. (b) Schematic representation of the alternative splice forms expressed in melanocyte and brain tissues. (c) Deletion 871-3310 in PB's MYO5A gene disrupts the F-exon as well as its $5^{\prime}$ and $3^{\prime}$ flanking intronic sequences. Number 1 of the base pair corresponds to the first base of the $5^{\prime} \mathrm{F}$-intron sequence. PCR amplification of the E- and F-exons in PB and control (C) is shown. Primer pairs used (indicated by arrows for the F-exon) were previously described (9).

F-exon $(9,30)$. Thus, although patients' cells were not available to test the absence of the longest F-exon-containing MYO5A isoform, the deletion identified in patient $\mathrm{PB}$ predicts an exclusion of the F-exon in the final MyoVa product.

\section{Discussion}

A hallmark of Griscelli syndrome is hair hypopigmentation characterized by a silver-gray sheen and the presence of large clusters of pigment unevenly distributed in the hair shaft. Either a primary neurological impairment or immune abnormalities are associated with this GS phenotype. They result from two different gene defects underlying GS1 and GS2, respectively $(2,3)$. Mutations in the gene that encodes the molecular motor protein MyoVa cause GS1 and the dilute mutant phenotype in mice, whereas mutations in the gene that encodes Rab27a are responsible for GS2 and the ashen mouse model. We show herein that, in addition to these two previously

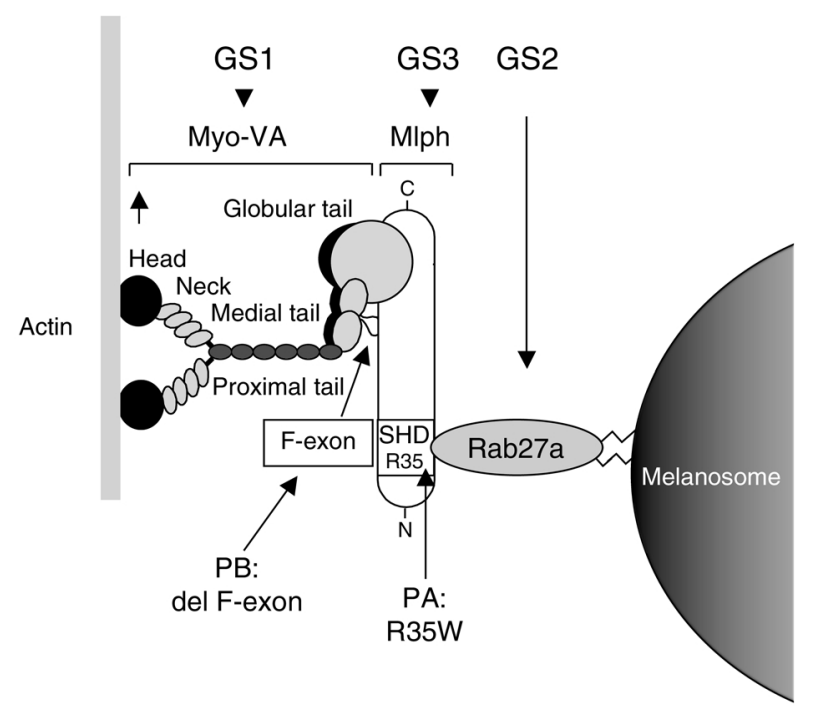

Figure 7

Scheme of the heterotrimeric protein complex involved in human melanosome transport. A defect in any of the proteins, MyoVa, Rab27a, or Mlph, leads to identical pigmentary dilution, found in the three forms of GS. The F-exon of MyoVa is required for MyoVa-Mlph interaction and the SHD of Mlph for Mlph-Rab27a interaction. The locations of the genetic defects identified in PA and PB are shown. 
described GS forms, a third genetically defined GS form (GS3) results from mutation in $M L P H$. Phenotypic expression of GS3 is restricted to the characteristic hypopigmentation of this syndrome and appears to be the human counterpart to the leaden mutant in mice (14).

GS3-associated albinism was found to be indistinguishable from that described in GS. Absence of linkage of this phenotype to the GS1-2 locus and its compatibility with the human chromosome region synthenic to the leaden locus designated $M L P H$ as a potential candidate gene. The missense mutation, identified in PA's MLPH gene, clearly affects the function of the corresponding encoding protein. The Mlph mutant protein is indeed unable to associate with Rab27a, either transiently overexpressed or endogenously expressed in melanocytes. The absolute requirement of an arginine residue at position 35 of the Mlph protein was also demonstrated by the complete failure to restore Mlph-Rab27a association when several residues, with molecular characteristics similar to those of arginine, were introduced at the same position. Mutation identified in this first GS3 case thus points to a critical residue of Mlph involved in direct interaction with Rab27a. In the absence of the tripartite protein complex (Rab27a-Mlph-MyoVa) formation in melanocytes (Figure 7), melanosomes cannot be connected to the actin network and thus transported toward the melanocyte tips. A previous in vitro study has shown that MyoVa interacts with Mlph through its F-exon (15). The study performed with cells from PB shows that this molecular interaction also operates in vivo in humans and is essential for melanosome transport. In contrast, the short F-exonnegative isoform of MYO5A appears sufficient to allow normal neurological development and function, since, unlike in previous patients identified with null mutation of $M Y O 5 \mathrm{~A}$, deletion of the F-exon in PB did not result in any neurological manifestations. A previous study in mutant mice in which Myo5a mutations failed to incorporate the F-exon led to similar conclusions (29).

Three genetic forms of GS have thus been identified. They result from mutations in MYO5A, $R A B 27 A$, and $M L P H$, respectively. The different phenotypes observed in each GS form are in accordance with the functions and tissue expression of the respective proteins (Figure 7): (a) The common pigmentary defect observed in GS1, GS2, and GS3 results from the absolute requirement and interaction of the three encoded proteins for melanosome transport, through the SHD of Mlph and the F-exon of MyoVa. (b) Normal neurological development and functions require the presence of $\mathrm{MyoVa}$, at least in its short F-exon-spliced isoform. Absence of MyoVa accounts for the primary severe neurological impairment observed in GS1. (c) The defective cytotoxic activity that characterized the GS2 phenotype results from the crucial role of Rab27a in the exocytosis of cytotoxic granules in $\mathrm{T}$ and NK lymphocytes (3), while MyoVa and Mlph are not expressed in cytotoxic cells (17). The severe and early immune dysregulation known as HS, which invariably leads to death in GS2 patients, most likely results from this defective cytotoxic activity. We indeed previously demonstrated that perforin mutations, which also impair this cytotoxic pathway, lead to the occurrence of an identical HS in patients with familial hemophagocytic lymphohistiocytosis (7). It is interesting to note that the same genetic defect in ashen mice is not associated with such severe immune manifestations. Although ashen mice exhibit a similar defective cytotoxic activity, their survival and fertility are identical to those of WT mice in nonprotected animal facilities, and they do not develop spontaneous HS. Similarly, perforin-deficient mice do not spontaneously develop HS (31). It will be interesting to investigate whether or not ashen mice develop HS when challenged with lymphocytic choriomeningitis virus, as perforin-deficient mice have been reported to do $(32,33)$.

Since the first description, by Griscelli et al., of a syndrome associating partial albinism and immunodeficiency (1), various expressions and three genetic causes have been associated with the typical hypopigmentation. GS diagnosis thus depends on the observation of this characteristic hypopigmentation in association with additional features. The molecular understanding of GS conditions now allows better prediction of the phenotypic consequences of any new anomalies identified in these three genes. Since prognosis, treatment, and genetic counseling differ considerably among the various forms, the performance of accurate genetic diagnosis, early in life, would be an important tool in medical decision-making.

The various genetic defects associated with GS have demonstrated that, although melanosome transport in humans involves a Rab27a-dependent, Mlphdependent, F-exon-MyoVa-dependent pathway, neither Mlph nor the F-exon of MyoVa plays a significant role in other functions. The existence of immune and neuronal cell type-specific effectors for MyoVa and Rab27a has thus been predicted. Forthcoming molecular and functional studies should show new partners of these proteins that are dedicated to organelle transport in these tissues.

\section{Acknowledgments}

We thank the patients involved in the study and their families for their cooperation. We are grateful to Ayse Metin for providing hair shaft samples from the patients, as well as Stéphanie Certain and Nathalie Lambert for excellent technical assistance. This work was supported by grants from INSERM, l'Association de Recherche sur le Cancer, and l'Association Vaincre les Maladies Lysosomales. 
1. Griscelli, C., et al. 1978. A syndrome associating partial albinism and immunodeficiency. Am. J. Med. 65:691-702.

2. Pastural, E., et al. 1997. Griscelli disease maps to chromosome $15 \mathrm{q} 21$ and is associated with mutations in the myosin-Va gene. Nat. Genet. 16:289-292.

3. Ménasché, G., et al. 2000. Mutations in RAB27A cause Griscelli syndrome associated with hemophagocytic syndrome. Nat. Genet. 25:173-176.

4. Bahadoran, P., et al. 2001. Rab27a. A key to melanosome transport in human melanocytes. J. Cell Biol. 152:843-850.

5. Langford, G.M., and Molyneaux, B.J. 1998. Myosin V in the brain: mutations lead to neurological defects. Brain Res. Brain Res. Rev. 28:1-8.

6. Blanche, S., et al. 1991. Treatment of hemophagocytic lymphohistiocytosis with chemotherapy and bone marrow transplantation: a singlecenter study of 22 cases. Blood. 78:51-54.

7. Stepp, S., et al. 1999. Perforin gene defects in familial hemophagocytic lymphohistiocytosis. Science. 286:1957-1959.

8. de Saint Basile, G., and Fischer, A. 2001. The role of cytotoxicity in lymphocyte homeostasis. Curr. Opin. Immunol. 13:549-554.

9. Pastural, E., et al. 2000. Two genes are responsible for Griscelli syndrome at the same 15q21 locus. Genomics. 63:299-306.

10. Jenkins, N.A., Copeland, N.G., Taylor, B.A., and Lee, B.K. 1981. Dilute (d) coat colour mutation of DBA/2J mice is associated with the site of integration of an ecotropic MuLV genome. Nature. 293:370-374.

11. Wilson, S.M., et al. 2000. A mutation in Rab27a causes the vesicle transport defects observed in ashen mice. Proc. Natl. Acad. Sci. U. S. A 97:7933-7938

12. Stinchcombe, J.C., et al. 2001. Rab27a is required for regulated secretion in cytotoxic t lymphocytes. J. Cell Biol. 152:825-834.

13. Haddad, E.K., Wu, X., Hammer, J.A., and Henkart, P.A. 2001. Defective granule exocytosis in rab27a-deficient lymphocytes from ashen mice. J. Cell Biol. 152:835-842.

14. Matesic, L.E., et al. 2001. Mutations in Mlph, encoding a member of the Rab effector family, cause the melanosome transport defects observed in leaden mice. Proc. Natl. Acad. Sci. U. S. A. 98:10238-10243.

15. Wu, X.S., et al. 2002. Identification of an organelle receptor for myosinVa. Nat. Cell Biol. 4:271-278.

16. Strom, M., Hume, A.N., Tarafder, A.K., Barkagianni, E., and Seabra, M.C. 2002. A family of Rab27-binding proteins. Melanophilin links Rab27a and myosin Va function in melanosome transport. J. Biol. Chem. 277:25423-25430.

17. Hume, A.N., et al. 2002. The leaden gene product is required with
Rab27a to recruit myosin Va to melanosomes in melanocytes. Traffic. 3:193-202.

18. Sanal, O., et al. 2002. Griscelli disease: genotype-phenotype correlation in an array of clinical heterogeneity. J. Clin. Immunol. 22:237-243.

19. Barrat, F., et al. 1996. Genetic and physical mapping of the ChédiakHigashi syndrome on chromosome 1q42-43. Am. J. Hum. Genet. 59:625-632.

20. Dib, C., et al. 1996. A comprehensive genetic map of the human genome based on 5,264 microsatellites. Nature. 380:111-135.

21. Menasché, G., et al. 2003. Biochemical and functional characterization of Rab27a mutations occurring in Griscelli syndrome patients. Blood. 101:2736-2742.

22. Kuroda, T.S., Fukuda, M., Ariga, H., and Mikoshiba, K. 2002. The Slp homology domain of synaptotagmin-like proteins 1-4 and Slac2 functions as a novel Rab27A binding domain. J. Biol. Chem. 277:9212-9218.

23. Seraphin, B., et al. 1996. An efficient PCR mutagenesis strategy without gel purificiation step that is amenable to automation. Nucleic Acids Res. 24:3276-3277.

24. Venter, J.C., et al. 2001. The sequence of the human genome. Science. 291:1304-1351.

25. Fukuda, M. 2002. The synaptotagmin-like protein (Slp) homology domain 1 of Slac2-a/melanophilin is a critical determinant of GTPdependent, specific binding of Rab27A. J. Biol. Chem. 277:40118-40124.

26. Ostermeier, C., and Brunger, A.T. 1999. Structural basis of Rab effector specificity: crystal structure of the small $\mathrm{G}$ protein Rab3A complexed with the effector domain of rabphilin-3A. Cell. 96:363-374.

27. Wu, X., et al. 2001. Rab27a enables myosin Va-dependent melanosome capture by recruiting the myosin to the organelle. J. Cell Sci. 114:1091-1100.

28. Huang, J.D., et al. 1998. Molecular genetic dissection of mouse unconventional myosin-VA: head region mutations. Genetics. 148:1951-1961.

29. Huang, J.D., et al. 1998. Molecular genetic dissection of mouse unconventional myosin-VA: tail region mutations. Genetics. 148:1963-1972.

30. Lambert, J., Naeyaert, J.M., Callens, T., De Paepe, A., and Messiaen, L. 1998. Human myosin V gene produces different transcripts in a cell type-specific manner. Biochem. Biophys. Res. Commun. 252:329-333.

31. Kagi, D., et al. 1994. Cytotoxicity mediated by T cells and natural killer cells is greatly impaired in perforin-deficient mice. Nature. 369:31-37.

32. Matloubian, M., et al. 1999. A role for perforin in downregulating T-cell responses during chronic viral infection. J. Virol. 73:2527-2536.

33. Kagi, D., Odermatt, B., and Mak, T.W. 1999. Homeostatic regulation of CD8+ T cells by perforin. Eur. J. Immunol. 29:3262-3272. 